\title{
Editorial
}

\section{Improvisation or planning the control of an infectious disease outbreak?}

Chile has more than 6,000 commercial cattle dairy producers, which are mainly located in Los Rios and Los Lagos regions. The country has one million hectares of grassland, 500,000 dairy cows and many dairy plants including some multinationals. Nevertheless, this productive sector faces certain animal-health problems which undermine the production, economic return, and potentially limit international trade. Additionally, they diminish the overall health of the herds, and some of them also represent a public health hazard.

Livestock handlers, veterinarians, abattoir workers, meat inspectors, laboratory staff handling biological samples from infected cattle, people consuming unpasteurized milk or other dairy products and improperly prepared meats, infants, the elderly, the immunocompromised, and those with other underlying health conditions are at increased risk of contracting bovine zoonotic infections.

Extensive outbreaks of zoonotic disease are not uncommon in Chile, as seen recently in southern Chile due to an outbreak of human Q fever. The perception of the general public regarding the risks involved considerably influences policy-making in the health field. In this regard, the WHO makes the following recommendations to deal with emerging diseases:

$\square$ New mechanisms of surveillance and response are required, using new tools (satellite remote sensing data, analytical molecular epidemiology) and bringing together different disciplines (medical, veterinary, population biology, information technology, diagnosis). $\square$ Integrating the early warning and alert systems of international organizations (WHO, FAO and OIE) to facilitate early detection of potentially linked animal and public health events.

$\square$ Integrating animal and human health data at national and regional levels, including an inter-sectoral committee for zoonosis preparedness and control.

Perhaps, this may be a good opportunity to propose a zoonosis health network (at least at a regional base) in order to manage outbreaks in the future with the structure and level of information recommended by the WHO.

Editorial Committee Austral Journal of Veterinary Sciences 